\title{
Ueber $\beta$-Digitoxin.
}

\author{
Von H. Kiliani.
}

(Eingegangen den 9. VI. 1895.)

Das reine Digitalinum verum zeichnet sich vor allen bisher in den Handel gebrachten Digitalispräparaten nach den Versuchen des Herrn Prof. B o ehm dadurch aus, dais es nur die typische Wirkung auf das Herz, aber keinerlei schädliche Nebenwirkung veranlafst. Trotz dieses grofsen Vorzuges vermochte es bisher nicht recht Eingang in die ärztliche Praxis zu gewinnen, weil von klinischer Seite wiederholt Mitteilungen gemacht wurden, wona ch sich mit dem Digitalinum verum doch nicht immer die gleichen Heilerfolge erzielen lassen wie mit dem althergebrachten Infusum Digitalis. Herr Prof. B o ehm sprach deshalb mir gegenüber die Vermutung aus, dafs in den Blättern der Digitalis purpurea noch irgend ein besonderes Herzgift stecken müsse, und er veranlafste mich, auch die pharmakologisch wichtigen Bestandteile der Blätter einer erneuten Untersuchung zu unterwerfen, indem er sich zugleich bereit erklärte, die nötig werdenden Tierversuche auszutühren. Für diese Anregung sowie für die äulserst wertvolle Förderung der Arbeit durch zahlreiche pharmakologische Experimente bin ich Herrn Prof. Boohm zu lebhaftestem Danke verpflichtet, denn die Untersuchung führte mehrfach zu sehr überraschenden Ergebnissen, vor allem aber zu dem wichtigen Hauptresultate, dafs die aus den Blattern gewonnenen Glykoside völlig verschiedensind vonjenenansden Samen. Das Digifonin, welchessichin letzterensoreichlich vortindet, konnte bisherinden Blätern überhaupt nicht a ufgefunden werden, ebensowenig das Digitalinum verum. Andererseits aber enthalten die in üblicher Weise dargestellten Samenglykoside kein Digitoxin.

Heute soll nur über einen Teil der hisher erhaltenen Resultate berichtet werden und zwar über die Gewinnung und die Eigenschaften eines Herzgiftes, welches entweder identisch oder zum mindesten nahe verwandt ist mit $\mathrm{S}$ c h mi edeberg's Digitoxin. 
Da ich von vornherein bei der Untersuclsung der Blätter einen möglichst vollständigen Ueberblick über alle Extraktivstoffe gewinnen wollte, wurden die grob zerstofsenen Blätter zuerst zweimal mit Wasser extrahiert, dann wieder möglichst rasch an der Luft getrocknet und hierauf mit 50prozentigem Alkohol ausgezogen. Jeder der beiden so gewonnenen Extrakte wurde für sich untersucht.

Die mit Wasser befenchteten Blätter besitzen bekanntlich aufserst grofse Neigung zur Schimmelbildung; ich habe deshalb anfangs das Wasser vor seiner Verwendung mit Chloroform geschüttelt, aber bald gefunden, das dies nicht immer hilft. Dagegen kann jenem Uebelstande leicht und sicher dadurch abgeholfen werden, dafs man das Wasser mit 5 Proz. seines Gewichtes 95 prozentigen Alkohols versetzt. Man nimmt auf 1 Teil Blätter 3 Teile dieses Extraktionsmittels, sorgt für gleichmälsige Mischung und läfst 12 Stunden unter Schutz vor Verdunstung stehen. Selbst bei zweitägiger Digestion gehen, wie besondere Versuche lehrten, nicht mehr Extraktivstoffe in Lösung als innerhalb jener kurzen Zeit. Durch Auspressen der Masse wurden aus $1 \mathrm{~kg}$ Blätter regelmälsig 2400 bis $2500 \mathrm{~g}$ rotbraunen Extrakts gewonnen. Der Rückstand wird zum zweiten Male mit der gleichen Menge Lösungsmittel behandelt, das zweite, äufserst verdünnte Extrakt aber nur zum Ansetzen neuer Blatter verwendet. Der erste Extrakt wurde in Flaschen gegossen, ca. 3 Stunden ruhig stehen gelassen, damit der beim Eingiefsen entstandene starke Schaum verschwindet und nun die Flasche völlig mit Aether aufgefüllt. Schüttelt man dann mehrmals um, so findet infolge der Absorption von Aether durch das Wasser eine Volumenabnahme statt, welche durch neuen Aetherzusatz wieder ausgeglichen wird, und auf diese Weise d. h. durch möglichsten Ausschlufs der Luft ist es möglich, die bei Gegenwart von Luft aufserordentlich zum Schäumen und zur Emulsion geneigte wässrige Lösung ohne Schwierigkeit mit Aether zu extrahieren. Dieser färbt sich tief grün; man wiederholt die Operation 3-4 mal, bis der letzte Auszng nur mehr schwach grün erscheint. Von der Untersuchung der verbleibenden wässrigen Lösung wird in einer späteren Abhandlung die Rede sein; bier soll nur über den Aether-Auszug berichtet werden. Destilliert man den Aether direkt ab, so erhält man einen tiefgrünen Sirup, aus dem auf keinerlei Weise eine Krystallisation 
zu erzielen ist. Schüttelt man aber die ätherische Lösung zuror mit sehr verdünnter Sodalösung, so nimmt diese unter starker Rotfärbung eine ziemlich grolse Menge von organischer Substanz zugleich mit dem grölsten Teile des in den Aether übergegangenen Alkohols (aus dem Extraktionsmittel stammend) auf, und lälst man dann den Aether nach sorgfültiger Trennung von der alkalischen Flüssigkeit 12-24 Stunden im bedeckten Gefäfse stehen, so bilden sich an den Gefäfswänden, namentlich beim Reiben, kleine grünweifse Wärzchen, welche an und für sich in reinem Aether so gut wie unlöslich sind und in den Aetherauszug nur durch die Vermittlung der grofsen Menge harziger Substanz und des Alkohols tibergegangen waren. Der von denselben abgegossene Aether wird nun destilliert, wobei sich allmählich immer mehr von jener Substanz in Krusten ablagert. Die aus $1 \mathrm{~kg}$ Blătter gewonnene ätherische Lösung wird bis $\mathrm{zu}$ einem Volumen von ca. $10 \mathrm{ccm}$ konzentriert, dann einige Stunden, geschützt vor Verdunstung, stehen gelassen and endlich von der Kruste abgegossen. ${ }^{1}$ ) Letztere wäscht man zweimal durch Decantieren mit Aether. Aus $1 \mathrm{~kg}$ Blätter erhält man so regelmälsig $0,15 \mathrm{~g}$ Rohprodukt. In weit reichlicherer Menge ist der Körper aus dem mittels 50 prozentigem Alkohol bereiteten Extrakte der vorher mit Wasser erschöpften und wieder lufttrocken gewordenen Blätter in folgender Weise zu gewinnen:

Je $1 \mathrm{~kg}$ dieses Materials wird mit $3 \mathrm{~kg} 50$ prozent. Alkohol 12 Stunden digeriert, das abgeprefste stark grüne Extrakt unter energischem Umrühren mit $0,4 \mathrm{~kg}$ Liquor Plumbi subacet. versetzt und nach ca. 2 Stunden filtriert. Der äufserst voluminöse schleimige Niederschlag schliefst auch nach vollständigem Abtropfenlassen auf dem Filter noch sehr erhebliche Mengen von Extrakt ein, welche man leicht durch Abnutschen gewinnen kann, wenn man dabei die Niederschlagsschichte auf der Nutsche immer nur mäfsig dick werden lä.sst, d. h. von Zeit zu Zeit den bereits ausgesaugten Niederschlag entfernt. Das Filtrat wird nun durch Verdampfung im Vakuum ${ }^{2}$ )

1) Diese Autterlauge enthält minimale Mengen eines zweiten, gut krystallisierenden Kơrpers, dessen Gewinnung aber nur bei Verarbeitung von mindestens $40 \mathrm{~kg}$ Bläitern moglich ist.

2) Zum raschen Eindampfen grofserer Quantitäten von Lösungen, welcbe leicht zersetzliche Substanzen enthalten, eignet sich ganz vorzüglich der Apparat von S o x h 1 e t (Chem. Ztg. 1894, I, i21), den ich nach vielf'ältiger Erprobung allen Fachgeiossen auf's Wärmste em. 
vom gröfsten Teile des Alkohols befreit, bis das schliefslich auftretende änfserst starke Schäumen die Fortsetzung der Operation unmöglich macht. Die konz. Lösung wird sodann in gleicher Weise wie das wässrige Extrakt 3-4 mal mit Aether und dieser behufs Befreiung von Alkohol nur mit Wasser (nicht mit Soda) geschüttelt. Die gewonnene ätherische Lösung ist in diesem Falle so reich an der krystallisierbaren Substanz, dafs häufig gofort nach ihrer Bebandlung mit Wasser die Abscheidung der grünweifsen Krusten beginnt, ganz besonders wenn man sie niedriger Temperatur aussetzt. Man giefst dann ab, destilliert, wobei immer stärkere Krustenbildung erfolgt, und läfst die von den Krystallen abgegossene, konzentrierte, tief grüne Lösung noch etwas in flacher Schale verdunsten, um innerhalb mehrerer Tage eine weitere Krystallisation zu erhalten, die allerdingz zumeist selbst stark grün gefärbt ist, aber noch ein bedeutendes Gewicht repräsentiert, so dafs man auf diesem Wege im ganzen aus $1 \mathrm{~kg}$ Blättern bei aufeinanderfolgender Behandlung derselben mit Wasser und 50 prozentigem Alkohol nahezu $1 \mathrm{~g}$ dieser leicht krystallisierbaren Substanz gewinnt.

Nachdem dies festgestellt war, lag es nahe zu versuchen, ob man nicht das gleiche Resultat erhalten könnte, wenn man die Blätter direkt mit 50 prozentigem Alkohol extrahiert. Dabei stellt sich aber, wohl in Folge der grofsen Masse vou ölig.harzigen Stoffen, welche sofort in die Lösung und dann in den Aether übergehen und in diesem grölstenteils verbleiben, anch wenn man mit Soda

pfehlen kann. Namentlich wenn man die Vorlage durch Eis oder Kältemischung energisch kühlt, erfolgt die äufserst rasch vorsichgohende Verdampfung nahezu bei Zimmertemperacur. Das von Soxhlet benutzte Quecksilbermanometer habe ich an meinem Apparate mit Vorteil durch ein direkt an der $K$ ö $r$ ti n g'schen Pumpe betestigtes Metallmanometer orsetzt.

Während man im Allgemeinen bei der Benutzung des Apparates die einzudampfende Lösung kontinuierlich in denselben einsaugen lälst, in demselben Mal'se als die Verdampfung fortschreitet, ist dies speziell bei den 50 Proz. alkoholischen Digitalisextrakten unmöglich. Sobald nämlich deren Alkoholgehalt durch die Destillation unter eine gewisse Grenze gesunken ist, beginnt ein so starkes Schäumen, dals ein F́lüssigkeitsvolumen von ca. $500 \mathrm{ccm}$ einen Raum von 7-8 Litern mit grolson Blasen erfủllt. Man bringt deshalb in diesem besonderen Falle sofort mehrere Liter Extrakt in den Kolben, stellt in diesen zur Erleichterung der Dampfblasenentwicklung einen feinen Holzstab und destilliert bis zu der oben angebenen Grenze. 
schüttelt, die Ausbeute wesentlich niedriger, sie beträgt aber immerbin noch ca. $0,5 \mathrm{~g}$ pro $1 \mathrm{~kg}$ Blätter.

Zur Reinigung des Rohproduktes kann man zwei Wege einschlagen :

Entweder löst man es bei gewöhnlicher Temperatur in einem Gemisch gleicher Volumina Methylalkohol und Chloroform (35 Gew.-T. Methylalkohol und 65 Gew.-T. Chloroform) und setzt dann Aether $(0,72)$ hinzu, bis höchstens ein leichtes Opalisieren, keinenfalls aber ein bleibender Niederschlag entsteht, wozu etwa das halbe Gewicht des Methylalkobol-Chloroforms genügt. Nach kurzer Zeit beginnen sich bübsche kleine Prismen in dichten Krusten abzuscheiden, welche in gleicher Weise weiter gereinigt werden können, wobei ein Schütteln der ursprünglichen Methylalkohol-Chloroform-Lösurg mit Blutkoble sehr förderlich wirkt.

Oder man verwendet als Lösungsmittel 85 prozentigen Alkohol und zwar 5 Gewichts-Teile auf 1 Teil Rohprodukt. Bei anhaltendem Kochen erfolgt vollständige Lösung, welche man hier durch $\mathrm{K}$ o c h e mit Blutkohle reinigt. Beim Erkalten bilden sich langsam weifse und sobald die Substanz wirklich rein ist, ganz farblose Warzen von blättrigen Krystallen. Das Umkrystaliisieren mufs nach gleichem Prinzip mehrmals wiederholt werde:ı unter allmählicher Steigerung der Menge des Lösungsmittels bis auf 10 Gewichts-Teile pro 1 Teil Jufttrockene Substanz.

Die aus Methylalkohol-Chloroform gewonnenen Krystalle aind wasserfrei, die aus der 85 prozentigen alkoholischen Lösung abgeschiedenen enthalten Krystallwasser. Recht merkwürdig ist die $\mathrm{Be}$ obachtung, dafs die ersteren bei $240^{\circ}$ noch fest oder höchstens schwach gesintert sind, wogegen die letzteren immer zwischen 145 und $150^{\circ}$ erweichen. Durch Auflösung in kochendem 85 prozentigem Alkohol lassen sich aber die wasserfreien Krystalle sofort wieder in wasserhaltige von der Erweichungstemperatur $145-150^{\circ}$ verwandeln.

In Wasser ist die reine Substanz nur spurenweise löslich. Bringt man einige Stänbchen davon in ca. $10 \mathrm{ccm}$ englische Schwefelsäure, so tritt allmählich eine charakteristische Rotfärbung ein, welche man etwa mit der sog. "weinroten“ Färbung der Lackmustinktur durch Kohlensäure vergleichen könnte; die Färbung wird durch Znsatz eines Tropfens verdünnten Bromwassers verstärkt, steht aber 
bezäglich ihrer Intensität weit zurück gegen die analoge Farbenreaktion des Digitalinum verum. In konz. Salzsäure löst sich die Substanz zunächst mit gelber Farbe, dann tritt ein ganz charakteristisches Opalisieren ein und allmällich wird die Lösung intensiv grün.

Die Analysen des mittelst 85 prozentigem Alkohol gereinigten Materials gaben folgende Werte:

I. $0,3756 \mathrm{~g}$ lufttrockener Substanz verloren im Vakuum sehr rasch $0,0483 \mathrm{~g}$ und dann bei $105^{\circ}$ noch $0,0061 \mathrm{~g}$, im Ganzen $0,0544 \mathrm{~g} \mathrm{H}_{2} \mathrm{O}$.

II. $0,1404 \mathrm{~g}$ bei 1050 getrockneter Substanz lieferten $0,3195 \mathrm{~g}$ $\mathrm{CO}_{2}$ und $0,1096 \mathrm{~g} \mathrm{H}_{2} \mathrm{O}$.

IXI. $0,1621 \mathrm{~g}$ ebenso $0,367 \mathrm{ig} \mathrm{CO}_{2}$ und $0,1230 \mathrm{~g} \mathrm{H}_{2} \mathrm{O}$.

Berechnet für $\mathrm{C}_{28} \mathrm{H}_{46} \mathrm{O}_{10}+5 \mathrm{H}_{2} \mathrm{O}$ :

$$
\mathrm{H}_{2} \mathrm{O} \quad 14,24
$$

Berechnet fur $\mathrm{C}_{28} \mathrm{H}_{46} \mathrm{O}_{10}$ :

C $\quad 61,99$

H $\quad 8,49$
Getunden:

14,48

Gefunden:

II. III.

$62,06 \quad 61,78$

$8,67 \quad 8,43$

Nuchdem das gleiche Material 8 Tage lang an der Luft gelegen hatte, fand ich nurmehr 12,3 Proz. Wasser, es scheint also ganz langsame Verwitterung stattzufinden.

Die beschriebene Substanz ist ein Glykosid: Erhitzt man sie nur wenige Minuten mit verdünnter Salzsäure in kochendem Wasser, so entsteht ein gelbes Harz und die von diesem abfiltrierte $L$ sung verursacht reichliche Reduktion von $F$ ehling's Reagens.

Alle diese Beobachtungen sowie auch die ersten Versuche, welche Herr Prof. B o e hm (schon im April 1894) mit meinen "Krystallen aus Aether" ausführte, schienen anzudeuten, dafs letztere mit keinem der bisher bekannten Digitalisabkömmlinge identisch seien. Herr Prof. Bo $\theta \mathrm{hm}$ schrieb mir am 20. April 1894 sogar direkt: "Sie haben also ohne Frage ein neues Digitalisglykosid entdeckt." Erst als es mir trotz aller Mühe und Sorgtalt absolut nicht gelingen wollte, aus den Blättern einen Körper zu isolieren, welcher $k$ e in Glykosid war und zugleich die Eigenschaften von $\mathrm{S} \mathrm{c} \mathrm{h} \mathrm{mi} \mathrm{ed} \mathrm{e-}$ berg's Digitoxin gezeigt hätte, tauchten in jener Richtung Zweifel auf. Ich bezog dann von E. M erck in Darmstadt eine kleine Quantităt Digitoxin, welches laut Mitteilung jener Firma „zwar nach 
den Angaben Sch mi edeberg's dargestellt ist, dessen Schmelzpunkt jedoch nicht damit übereinstimmt"“. Zunächst stellte ich fest, dals auch das Merck'sche Präparat beim Erhitzen mit Säure Zncker abspaltet; die Erweichungstemperatur wurde ebenso wie bei meinen „Krystallen aus Aether" zu annähernd $145^{\circ}$ gefunden. Gegen englische Schwetelsäure verhält sich das M erck'sche Digitoxin etwas anders als meine reine Substanz, es giebt nämlich eine sehr schmutzige rote Färbung. Jedenfalls aber ist M e rck's Präparat noch nicht ganz einheitlich bezw. rein. Denn wenn man es in $10 \mathrm{~T}$. kochenden 85 prozentigen Alkohols auflöst, erhält man eine gel be Flüssigkeit und beim Erkalten scheiden sich deutlich zweierlei Krystalle aus, zuerst weifse kleine Wärzchen und dann kommen unverkennbar dieselben schönen farblosen Krystallblätter wie bei meinen reinen „Krystallen aus Aether". Ich habe dieses Gemisch von weilsen und farblosen Krystallen direkt analysiert und folgende Werte erhalten :

I. $0,4565 \mathrm{~g}$ verloren im Vakuum rasch $0,0682 \mathrm{~g}$, dann bei $105^{0}$ noch $0,0056 \mathrm{~g}$, im ganzen $0,0738 \mathrm{~g} \mathrm{H}_{2} \mathrm{O}$.

II. $0,1611 \mathrm{~g}$ bei $105^{\circ}$ getrockneter Substanz gaben $0,373 \mathbf{g} \mathrm{CO}_{2}$ und $0,1259 \mathrm{~g} \mathrm{H}_{2} \mathrm{O}$.

Gefunden: 16,16 Proz. $\mathrm{H}_{2} \mathrm{O}, 63,14$ Proz. C, 8,68 Proz. H Schmiedeberg fand: 63,60 $8,50, "$,

S ch m i ed e berg hatte seine Substanz aus absolutem Alkohol bezw. Chloroformalkohol gewonnen, also natürlich wasserfreies Material bekommen, was ich ja auch an meinen "Krystallen aus Aether" beobachtete. Ueber die Identität von Merck's Präparat mit Schmiedeberg's Digitoxin scheint mir nun nach obigem kein Zweifel zu bestehen, so dafs jedenfalls die Schlufsfolgerung berechtigt sein dürfte, dals auch $S \mathrm{chmi}$ edebor'g's Digitoxin e in Glykosid war. Dagegen läfst sich vorläufig nicht mit voller Bestimmtheit behaupten, dafs auch meine "Krystalle aus Aether" identisch sind mit Schmiedeberg's Digitoxin. Ich vermute zwar, dafs letzteres ebensowenig wie das Merck'sche Präparat eine völlig einheitlicho Substanz war. Denn Schmiedeberg's Darstellungsmethode, welche sich einfach auf die Schwerlöslichkeit des Digitoxins in Wasser gründet, macht die Wahrscheinlichkeit der Beimengung anderer schwer löslicher Stoffe jedenfalls weit 
gröfser als mein Aether-Extraktions-Verfahren, bei welchem nach allen meinen Beobachtungen nur dieses $\theta$ in $\theta$ Glykosid in den Aether übergeht. Um aber jede neue Verwirrung in der DigitalisLitteratur zu vermeiden, möchte ich vorschlagen, bis zur späteren völligen Aufklärung des Sachverhaltes das Schmiedeberg'sche Präparat als a-Digitoxin, meine, ,Krystalle aus A ether"dagegen als $\beta$-Digitoxin zu bezeichnen. Stellt sich denn in Zukunft heraus, dals $\mathrm{Schmiedeberg's} \mathrm{Produkt}$ als wesentlichen Bestandteil wirklich nur das von mir dargestellte chemische Individuum enthält, so macht es keine Schwierigkeit, die Präfixa $a$ und $\beta$ zu beseitigen. Ueber die pharmakologische Untersuchung des $\beta$-Digitoxins wird Herr Prof. Boehm selbst berichten.

Die Spaltung des $\beta$-Digit ox in s läfst sich mit Leichtigkeit schon bei gew öhnlich or Temperatur bewerkstelligen in folgender Weise:

Man ubergielst 1 Teil lufttrockenes $\beta$-Digitoxin mit 10 Teilen eines Gemisches von 8 Teilen 50 prozentigem Alkohol und 2 Teilen konz. Salzsäure $(1,19)$. Bei fleifsigem Umschwenken der vor Verdunstung geschützten Mischung löst sich das Glykosid in 1-2 Stunden völlig auf. Nach 24 Stunden, innerhalb welcher Zeit manchmal ohnedies schon eine geringe Abscheidung von Krystallen zu beobachten ist, verzetzt man die nur schwach gelbe Flüssigkeit mit Wasser bis zum leichten Opalisieren, worauf alsbald eine reichliche Krystallisation entsteht. ZurVollendung derselben lälst man 12 Stunden im kalten Raum stehen und saugt dann ab unter Benutzung von zuerst 20, dann 10 prozentigem Alkohol als Waschflíssigkeit. Das Filtrat wird mit Wasser verdünnt und drei mal mit Chloroform geschüttelt, bei dessen Verdunstung zunächst ein Sirup verbleibt. Dieser verwandelt sich aber durch kurzes Erwärmen mit wenig 50 prozentigem Alkohol ebenfalls in einen dicken Brei der schon erwähnten Krystalle.

Zur endgiltigen Reinigung des so gewonnenen $\beta-\mathrm{D}$ i g i t o $\mathrm{x}$ i $\mathrm{g} \theta \mathrm{n}$ ins genugt einmalige Auflösung in 5 Teilen warmen 95 prozentigen Alkohols, Schütteln der Lösung mit etwas Blutkohle und vorsichtige Sättigung des vơllig farblosen Filtrates mit Wasser. Man 
erhält auf diese Weise prächtige, relativ grolse Prismen (sehr häufig Darchkreuzungazwillinge).

Die reinen Krystalle geben keine Farbenreaktion mit Eisenchlorid, ebensowenig mit englischer Schwefelsäure oder mit konz. Salzsäure und unterscheiden sich dadurch wesentlich vom $\beta$ Digitoxin. Sie reagieren neutral und sind unlöslich in Natriumcarbonat. Mischt man die Krystalle mit letzterem Reagens und giebt Kaliumpermanganat hinzu so erfolgt wenigstens innerhalb kurzer Zeit keine Reduktion.

Das Digitoxigenin enthält keiu Krystallwasser und ist bei $220^{\circ}$ noch fest.

$0,1257 \mathrm{~g}$ vakuumtrocken Substanz lieferten $0,335 \mathrm{~g} \mathrm{CO}$ und $0,1023 \mathrm{~g} \mathrm{H}_{8} \mathrm{O}$.

$\begin{array}{ccc}\text { Berechnet für } \mathrm{C}_{21} \mathrm{H}_{32} \mathrm{O}_{\mathbf{1}}: & \text { Gefunden : } \\ \mathrm{C} & \mathbf{7 2 , 4 1} & \mathbf{7 2 , 6 8} \\ \mathbf{H} & \mathbf{9 , 1 9} & \mathbf{9 , 0 4}\end{array}$

Die weitere Untersuchung des sowohl chemisch als pharmakologisch interessanten Körpers werde ich möglichst bald in Angriff nehmen.

Zum Nachweise des bei der Spaltung entstandenen Z u ckers wurde die mit Chloroform extrabierte, ganz farblose wässerige Lösung mittelst Silberoxyd von der Salzsäure befreit und im Vakuum über Schwefelsäure bis zum Sirup verdunstet. Dieser verwandelte sich nach kräftigem Umrübren über Nacht in einen dicken Brei von relativ grolsen Krystallen, deren ganzer Habitus sofort erkennen Jiels, dals keinentalls ein dem allgemeinen Typus $\mathrm{C}_{6} \mathrm{H}_{18} \mathrm{O}_{6}$ angehöriger Zucker vorlag. Zufällig war ich durch andere Arbeiten verhinderts die Masse augenblicklich zu verarbeiten und lieis sie deshalb im Exsikkator über Schwefelsäure stehen. Als ich sie nach ca. 14 Tagen wieder vornahm, war zu meiner unliebsamen Ueberraschung ein wesentlicher Anteil der Krystalle unter Gelbfärbung schmierig geworden; es hatte also eine Zersetzung stattgefunden, deren Grund mir bisher unbekannt ist. Durch sofortiges Anrühren mit wenig Methylalkohol konnte ich deshalb leider nurmehr einen kleinen Teil der urspringlichen Krystalle retten, was um so bedauerlicher ist, als die einzige Elementaranalyse, welche ich aus obigem Grunde auszufübren vermochte, ein recht merkwüdiges Resultat ergab. 
$0,1888 \mathrm{~g}$ vakuumtrockener Substanz lieferten $0,3386 \mathrm{~g} \mathrm{CO}$ und $0,1452 \mathrm{~g} \mathrm{H}_{2} \mathrm{O}$.

$\begin{array}{ccc}\text { Berechnet fur } \mathrm{C}_{6} \mathrm{H}_{12} \mathrm{O}_{4}: & \text { Gefunden: } \\ \mathrm{C} & 48,65 & 48,91 \\ \mathrm{H} & 8,11 & 8,54\end{array}$

Hieraus kann man vorläufig nur schliefsen, dals bei der Spaltung des $\beta$-Digitoxins ein eigenartiger Zucker, die Digitoxose entsteht, deren Formel aber entschieden noch genauer kontrolliert werden mufs.

Der Zucker ist in Wasser leicht löslich; ein mittels Wasser bereiteter Sirup desselben liefert langsam grofse, schön ausgehildete Prismen. Die Digitoxose löst sich reichlich in Aceton; aus ihren alkoholischen Lösungen wird sie nur, wenn jene ganz konzentriert sind, durch Aether gefällt und zwar regelmälsig direkt als Krystallmehl. Diese Eigenschaften deuten auch schon darauf hin, dafs die Digitoxose weniger Sauerstoff enthält als der allgemeinen Formel $\mathrm{C}_{\mathrm{n}} \mathrm{H}_{2 \mathrm{n}} \mathrm{O}_{\mathrm{n}}$ entspricht.

Zum Schlusse sei noch bemerkt, dafs die Untersuchung der übrigen aus den Digitalisblättern gewinnbaren Glykoside schon ziemlich weit vorgeschritten ist und dafs ich z. B. schon seit einiger Zeit im Besitze von gut krystallisierten Glykosiden bin, deren Herzwirkung etwa viermal so stark ist als jene des $\beta$-Digitoxins. Ich hoffe hierüber in Bälde berichten zu können. 